Published in final edited form as:

A A Case Rep. 2017 April 15; 8(8): 213-215. doi:10.1213/XAA.0000000000000467.

\title{
Postoperative Pediatric Cerebellar Mutism after Posterior Fossa Surgery
}

\author{
Jerry Y. Chao, MD [Assistant Professor], \\ Department of Anesthesiology, Albert Einstein College of Medicine, Montefiore Medical Center, \\ Bronx, NY/USA \\ Che Liu, BS [Medical Student], \\ Department of Anesthesiology, Albert Einstein College of Medicine, Montefiore Medical Center, \\ Bronx, NY/USA, che.liu@med.einstein.yu.edu
}

Naveen Shetty, BA [Medical Student], and

Department of Anesthesiology, Albert Einstein College of Medicine, Montefiore Medical Center, Bronx, NY/USA, naveen.shetty@med.einstein.yu.edu

\section{Ushma Shah, MD [Clinical Instructor]}

Department of Anesthesiology, Rutgers University - Robert Wood Johnson University Hospital, New Brunswick, NJ 08901, ushma03@gmail.com

\begin{abstract}
Cerebellar mutism syndrome (CMS) is a common complication of posterior fossa surgery that can confound the post-anesthetic exam and have long lasting impacts. There is confusion surrounding its precise description, diagnostic features and associated morbidity. Here we discuss the most up to date knowledge of CMS drawing from a clinical case in the context of three new reports: (1) an international consensus paper presenting a new proposed working definition by the Iceland Delphi Group $^{1}$, (2) a knowledge update by Gadgil et al ${ }^{2}$ (3) and a review of neuroimaging-based data elucidating the etiology of CMS by Patay ${ }^{3}$.
\end{abstract}

\section{Keywords}

case report; postoperative cerebellar mutism; pediatric neuroanesthesia

Corresponding Author: Jerry Y. Chao, MD, Department of Anesthesiology, Albert Einstein College of Medicine, Montefiore Medical Center, 111 E 210 th St, Bronx, NY 10467, Phone: (718) 920-4316, Fax: (718) 881-2245, jerry.chao@med.einstein.yu.edu. Jerry Y. Chao, MD, Conflicts of Interest: None

Che Liu, BS, Conflicts of Interest: None

Naveen Shetty, BA, Conflicts of Interest: None

Ushma Shah, MD, Conflicts of Interest: None

Author contributions: Dr. Chao and Dr. Shah cared for the patient, gathered case information, and were involved in manuscript preparation. Mr. Liu and Mr. Shetty were involved with manuscript preparation. 


\section{Introduction}

A 7-year old child with a posterior fossa tumor was anesthetized for craniotomy and resection in the prone position. Surgery was complicated by postoperative cerebellar mutism syndrome (CMS). We present this case as a basis for discussing new developments in the definition, diagnosis and theorized mechanisms of postoperative CMS, a complication of posterior fossa surgery that can confound the post-anesthetic exam and have long lasting impact. Considerable confusion has surrounded the precise description of CMS, its diagnostic features and associated morbidity. In an international consensus statement in May 2016, the Iceland Delphi Group, a cohesive panel of clinicians and researchers from multiple relevant disciplines, presented a new working definition of $\mathrm{CMS}^{1}$. We highlight this new definition, a recent update of the features of CMS by Gadgil et al ${ }^{2}$ as well as work by Patay ${ }^{3}$ unraveling the pathophysiology, markers and possible etiology of CMS using magnetic resonance imaging. In doing so, we present the most recent knowledge of this clinical entity.

\section{Consent for publication}

Written informed consent for publication of this case report was obtained from the mother of the patient after she and the patient reviewed the case. All potential patient identifiers were carefully removed in the preparation of this manuscript.

\section{Case Description}

A 7-year old, $27 \mathrm{~kg}$ female patient presented with a one-month history of progressively worsening clumsiness and gait disturbance. Her parents reported she had headaches beginning two weeks prior to admission and nausea and non-bilious vomiting one week prior to admission. Neurological exam on admission demonstrated dysmetria as well as increased tone distributed in the bilateral arms and legs, right leg greater than left. Magnetic resonance imaging revealed a $5 \mathrm{~cm} \times 4 \mathrm{~cm} \times 3 \mathrm{~cm}$ midline lesion compressing the cerebellar vermis and underlying fourth ventricle consistent with a medulloblastoma. On the day prior to surgery an extraventricular drain was placed in the pediatric intensive care unit. In the operating room surgical exposure and tumor resection proceeded uneventfully in the prone position.

The surgical approach involved splitting the folia on the right upper cerebellar hemisphere and proceeding to a depth of $5 \mathrm{~mm}$ at which point tumor was encountered. Pathology confirmed the diagnosis of medulloblastoma. Careful dissection proceeded around the lesion after decompression with ultrasonic ablation (CUSA). The tumor extended to the level of the tentorium as well as to the floor of the fourth ventricle. The floor of the fourth ventricle was approached with the CUSA in low aspiration mode, gently delivering the tumor upwards. At no point was the floor of the fourth ventricle disturbed. Based on gross inspection complete resection of the tumor was achieved and hemostasis confirmed.

General anesthesia was induced with $30 \mathrm{mg}$ of lidocaine, $100 \mathrm{mg}$ of propofol, and $30 \mathrm{mg}$ of rocuronium. Total surgical time was approximately 6 hours with infusion times of 5 hours and 30 minutes for propofol and 5 hours 45 minutes for remifentanil. The propofol dosing ranged from 50 to $150 \mathrm{mcg} / \mathrm{kg} / \mathrm{min}$ and was completely discontinued 15 minutes before the 
end of surgery. The peak remifentanil infusion rate was $0.2 \mathrm{mcg} / \mathrm{kg} / \mathrm{min}$ and discontinued upon completion of the surgery and immediately prior to turning the patient to the supine position. Fentanyl was then was titrated to a respiratory rate of $15-20$ breaths per minute.

Upon emergence, the patient was hemodynamically stable, breathing spontaneously with greater than $8 \mathrm{~mL} / \mathrm{kg}$ tidal volumes off all pressure support, but unresponsive. Full train-offour on repeat neuromuscular testing was confirmed. The patient was stimulated by pinching the ears and nail beds and via sternal rub. She localized to pain but did not respond to commands. There was intermittent flexion and extension posturing of the upper extremities with generalization to tonic-clonic movements of the arms and legs. It is unclear if these movements represented a seizure or if the patient was post-ictal. She did not receive any antiepileptic medication perioperatively. These episodes occurred intermittently for two minutes and then stopped without treatment. The patient's mental status remained unchanged 30 minutes after all anesthetic administration was discontinued. She was transported to the radiology suite intubated and a head computerized tomography was performed that failed to show any significant intracranial findings that would explain her unresponsiveness. She was transported from radiology to the pediatric intensive care unit.

Three hours post procedure, she was extubated by the critical care team without incident. There were no further episodes of seizure-like movements. Despite being able to follow simple commands, the patient was nonverbal with slow and dysmetric movements. On postoperative day three she became more verbal, was able to follow more commands and was able to feed herself. However, movement and speech remained slowed. This has persisted months after surgery and she continues to undergo speech therapy and rehabilitation.

\section{Discussion}

Our clinical case serves as a basis for discussion of recent developments in clarifying the definition, diagnosis and mechanism of postoperative cerebellar mutism syndrome (CMS), an important consideration for the anesthesiologist. Historically, a variety of monikers including akinetic mutism, posterior fossa syndrome and cerebellar cognitive affective syndrome were used to describe the constellation of clinical findings after posterior fossa surgery. The inconsistent use of these terms has lead to confusion and difficulties in comparisons and interpretation across studies. Gudrunardottir et al recently reported in Childs Nerv Syst the results of an international consensus process whose aim was to refine and clarify terminology of clinical entities in the care of posterior fossa tumor patients ${ }^{1}$. The process involved the use of the modified nominal group technique, a procedure involving "multiple rounds of online Delphi questionnaires interspersed with a structured consensus conference with lectures, group work, and open discussion sessions." A new proposed working definition of cerebellar mutism was developed with draft recommendations for diagnosis and follow-up. Standardization of language will enhance clinical care and research endeavors to improve treatment and quality of life for those afflicted.

The new definition is as follows: 
"Post-operative pediatric CMS is characterized by delayed onset mutism/reduced speech $^{\mathrm{a}}$ and emotional lability after cerebellar or $4^{\text {th }}$ ventricle tumor surgery in children. Additional common features include hypotonia and oropharyngeal dysfunction/dysphagia. It may frequently be accompanied by cerebellar motor syndrome $^{\mathrm{b}}$, cerebellar cognitive affective syndrome $\mathrm{e}^{\mathrm{c}}$ and brain stem dysfunction including long tract signs ${ }^{\mathrm{d}}$ and cranial neuropathies. The mutism is always transient, but recovery from CMS may be prolonged. Speech and language may not return to normal, and other deficits of cognitive, affective and motor function often persist ${ }^{1} . "$

Gadgil et al published a knowledge update in Surgical Neurology International in March 2016 using two illustrative cases similar to our reported case ${ }^{2}$. They describe an 8-24\% incidence of CMS after posterior fossa tumor resection. On average, the onset of mutism occurs within 2 days but may not present until up to 7 days postoperatively. It may be difficult to diagnose in the immediate postoperative period given the altered mental status possible after delayed emergence from general anesthesia as well as the general timeline of CMS symptom onset. The duration of mutism averages 8 weeks with a range of a few days to as long as 5 months. The pathophysiologic mechanism is unknown but associated risk factors have been described from studies using regression methodology: (1) medulloblastoma is associated with a two to three fold increased risk, (2) vermal/midline location and high $4^{\text {th }}$ ventricle lesions are associated with increased risk compared to lateral locations and (3) tumor involvement or compression of the brainstem is associated with a higher risk. Larger tumor size may also be associated with higher risk but the data are conflicting.

Pathophysiologic mechanisms of CMS have been proposed by Patay in Childs Nerv Syst in July $2015^{3}$ from neuroimaging data. Damaged structures have been reasoned to be important in extrapolating the etiology CMS. The dentate-thalamo-cortical pathway, also known as the proximal efferent cerebellar pathway, is one possible pathway governing CMS. The circuitry of this pathway is as follows: cerebellar neurons form synapses in the dentate nucleus.

Subsequent neurons ascend and decussate, forming synapses at in the contralateral ventrolateral nucleus of the thalamus. From there, neurons innervate the motor and premotor cerebral cortices. This pathway serves as an "integration center" between the cerebral cortex and cerebellum and is responsible for motor activity, coordination and movement as well as modulating cognition and behavior. Efferent white matter damaged during tumor resection may lead to the hallmark symptoms of CMS. Bilateral damage to the dentate nuclei also seems to be necessary based on neuroimaging data. Hypertrophic changes observed in the olivary nucleus have also been found to be a fairly reliable surrogate imaging marker of

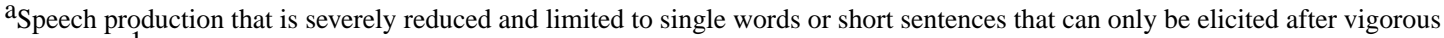
stimulation ${ }^{1}$.

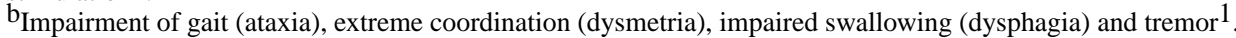

$\mathrm{c}^{\mathrm{A}}$ pattern of behavioral abnormalities that includes impairments of executive function (planning, set-shifting, abstract reasoning, verbal fluency, working memory), often with perseveration, distractibility or inattention; visual-spatial disorganization and impaired visual-spatial memory; personality changes with blunting of affect or disinhibited and inappropriate behavior; and difficulties with language production including dysprosodia, agrammatism and mild anomia ${ }^{1}$.

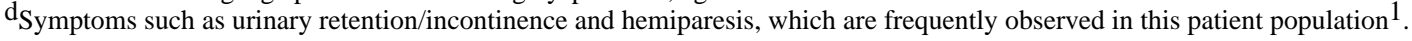


damage in retrospective studies. This imaging sign may be used as a future "validation tool" to diagnose CMS.

An important concept in the discussion of the pathophysiology of CMS is diaschisis, a "complex interplay between focal lesions, network dynamics, and changes in functional organization in remote areas ${ }^{3}$." CMS may be an example of diaschisis in which the function of the cerebral cortical speech areas is reduced as a result of damage to distant cerebellar structures sending efferent input. CMS as an entity highlights the important role of the cerebellum in executive function and the regulation of the higher order cognitive domains traditionally thought to be carried out in the cerebral cortex alone. The variable time to onset and recovery with subsequent potential long-term impairments that are the hallmarks of CMS make the theory of a one-time injury to neural pathways as the sole etiologic mechanism unlikely. Postoperative edema and vascular insufficiency due to injury or vasospasm may also be important.

Treatment for CMS consists of supportive care with the involvement of multidisciplinary teams for intensive inpatient rehabilitation (e.g. speech, physical and occupational therapy). However, there is no consensus on specific treatments. We were unable to find any studies investigating the association between anesthesia and CMS.

\section{Acknowledgments}

Funding: This manuscript was supported by NIH/National Center for Advancing Translational Science (NCATS) Einstein-Montefiore CTSA Grant Number UL1TR001073.

\section{References}

1. Gudrunardottir T, Morgan AT, Lux AL, Walker DA, Walsh KS, Wells EM, Wisoff JH, Juller M, Schmahmann JD, Keating RF, Catsman-Berrevoets C. Iceland Delphi Group. Consensus paper on post-operative pediatric cerebellar mutism syndrome: the Iceland Delphi results. Childs Nerv Syst. 2016; 32:1195-1203. [PubMed: 27142103]

2. Gadgil N, Hansen D, Barry J, Chang R, Lam S. Posterior fossa syndrome in children following tumor resection: knowledge update. Surg Neurol Int. 2016; 7(Suppl 6):S179-83. [PubMed: 27057398]

3. Patay Z. Postoperative posterior fossa syndrome: unraveling the etiology and underlying pathophysiology by using magnetic resonance imaging. Childs Nerv Syst. 2015; 31:1853-8. [PubMed: 26143277]

4. Pitsika M, Tsitouras V. Cerebellar mutism: A review. J Neurosurg Pediatrics. 2013; 12:604-14.

5. Robertson PL, Muraszko KM, Holmes EJ, Sposto R, Packer RJ, Gajjar A, Dias MS, Allen JC. Incidence and severity of postoperative cerebellar mutism syndrome in children with medulloblastoma: a prospective study by the Children's Oncology Group. J Neurosurgery. 2006; 105:444-51.

6. Di Rocco C, Chieffo D, Pettorini BL, Massimi L, Caldarelli M, Tamburrini G. Preoperative and postoperative neurological, neuropsychological and behavioral impairment in children with posterior cranial fossa astrocytomas and medulloblastomas: the role of the tumor and the impact of the surgical treatment. Child's Nerv Syst. 2010; 26:1173-1188. [PubMed: 20552208] 


\section{Table 1}

Risk factors for Cerebellar Mutism Syndrome (Gadgil et al ${ }^{2}$ )

\begin{tabular}{|l|l|l|}
\hline Significant Risk Factors & Possibly Significant Risk Factors & No Proven Significance \\
\hline Medulloblastoma Tumor Type & Pre-op Language Impairment & Gender \\
\hline Vermal/midline Tumor Location & Younger Age & Pre-op Hydrocephalus \\
\hline High $4^{\text {th }}$ Ventricle Location & Radical Resection & Post-op Meningitis \\
\hline Brainstem Involvement & Vermian Incision & Non-specialized Neurosurgeon \\
\hline & Large Tumor Size & Anesthetic technique \\
\hline
\end{tabular}

